\title{
A influência dos aplicativos de delivery de alimentos no hábito alimentar na região da grande São Paulo - SP
}

\author{
The influence of food delivery applications on food habits of the region of greater São \\ Paulo - SP
}
La influencia de las aplicaciones de entrega de alimentos en los hábitos alimentarios de la región de gran São Paulo - SP

Felipe Benite de Alcantara ${ }^{1 *}$, Giovanna Cavanha Corsi ${ }^{1}$, Carolina Moya Ramos Schubert ${ }^{1}$, Andrea Lorenzi $^{1}$, Ana Paula de Queiroz Mello ${ }^{1}$

\section{RESUMO}

Objetivo: Avaliar o uso dos Aplicativos de Delivery de Alimentos (ADAs) e a sua influência nos hábitos alimentares da população da grande São Paulo - SP. Métodos: Trata-se de um estudo transversal, desenvolvido na Grande São Paulo, entre 2019 e 2020, realizado com 307 indivíduos, de ambos os sexos, com idade a partir de 18 anos. Os dados foram obtidos através de um questionário online e as análises estatísticas feitas com o auxílio do programa Statistical Package for the Social Sciences ${ }^{\circledR}$. O valor de significância considerado foi de $p<0,05$. Resultados: Os dados sobre alimentação revelam um maior consumo dos fast foods $(p<0,001)$ e refrigerantes $(p<0,001)$ dos indivíduos que utilizam os ADAs com maior frequência. Além disso, a maior frequência de preparo de alimentos em casa está relacionada com a menor frequência de uso dos $\operatorname{ADAs}(p=0,046)$. Os indivíduos que são influenciados por mais de um tipo de propaganda $(p<0,001)$ e recebem cupons frequentemente $(p<0,001)$ costumam utilizá-los em maior proporção. Conclusão: $\mathrm{O}$ uso dos ADAs independe das características socioeconômicas e exerce uma influência negativa sobre os hábitos alimentares.

Palavras-chave: Aplicativos móveis, Comportamento alimentar, Consumo.

\begin{abstract}
Objective: To evaluate the use of Food Delivery Applications (FDAs) and their influence on the eating habits of the population of the greater São Paulo - SP. Methods: This is a cross-sectional study, developed in Greater São Paulo, between 2019 and 2020, conducted with 307 individuals, of both sexes, aged 18 years and over. The data were obtained through an online questionnaire and the statistical analyzes were carried out with the aid of the Statistical Package for the Social Sciences ${ }^{\circledR}$ program. The significance value considered was $p$ $<0.05$. Results: Food data reveal a higher consumption of fast foods $(p<0.001)$ and soft drinks $(p<0.001)$ of those who used FDAs more frequently. In addition, the higher frequency of food preparation at home is related to the lower frequency of use of FDAs $(p=0.046)$. Individuals who are influenced by more than one type of advertising $(p<0.001)$ and receive coupons frequently $(p<0.001)$ tend to use those in a greater proportion. Conclusion: The use of FDAs is independent of socioeconomic characteristics and has a negative influence on eating habits.
\end{abstract}

Key words: Mobile applications, Eating behavior, Intake.

\section{RESUMEN}

Objetivo: Evaluar el uso de Aplicaciones de Entrega de Alimentos (AEAs) y su influencia en los hábitos alimentarios de la población del Gran São Paulo - SP. Métodos: Se trata de un estudio transversal, desarrollado en Gran São Paulo, entre 2019 y 2020, realizado con 307 individuos, de ambos sexos, de 18 años y más. Los datos se obtuvieron a través de un cuestionario en línea y los análisis estadísticos se realizaron con la ayuda del programa Statistical Package for the Social Sciences $\AA^{\circledR}$. El valor de significancia considerado fue $p<0.05$. Resultados: Los datos alimentarios revelan una mayor frecuencia de consumo de comidas rápidas $(p<0,001)$ y refrescos $(p<0,001)$ de las personas que consumen AEA con mayor frecuencia.

${ }^{1}$ Centro Universitário São Camilo (CUSC), São Paulo - SP. *E-mail: nutrifelipebenite@gmail.com

SUBMETIDO EM: 1/2021

ACEITO EM: 1/2021

PUBLICADO EM: 2/2021 
Además, la mayor frecuencia de preparación de alimentos en el hogar se relaciona con la menor frecuencia de uso de AEA $(p=0,046)$. Las personas que se ven influenciadas por más de un tipo de publicidad $(p<0,001)$ y reciben cupones con frecuencia $(p<0,001)$ tienden a utilizarlos em mayor medida. Conclusión: El uso de AEAs es independiente de las características socioeconómicas y tiene una influencia negativa en los hábitos alimentarios.

Palabras clave: Aplicaciones móviles, Conducta alimentaria, Consumo.

\section{INTRODUÇÃO}

A alimentação do brasileiro vem passando por mudanças ao longo de pouco mais de 20 anos, visto que a contribuição calórica dos produtos prontos para o consumo aumentou e a dos alimentos in natura e minimamente processados diminuiu (MARTINS APB, et al., 2013). Em função disso, as taxas de brasileiros obesos aumentaram de $11,8 \%$ para $20,3 \%$ do ano de 2006 para 2019 (BRASIL, 2019).

De acordo com os dados da Pesquisa de Orçamentos Familiares (POF) 2017-2018, no Brasil, apesar da maior parcela dos gastos com alimentação ainda serem de refeições realizadas dentro de casa $(67,2 \%$ dos gastos totais), o consumo de alimentos fora do lar tem aumentado. Entre 2008-2009 e 2017-2018, o percentual de despesas com alimentação fora de casa nos gastos totais aumentou de $31,1 \%$ para $32,8 \%$, respectivamente (IBGE, 2019; IBGE, 2010).

Os locais que possuem maior frequência de aquisição de alimentos para consumo fora do lar são as lanchonetes e os restaurantes. As lanchonetes oferecem conveniência, praticidade e agilidade dos alimentos, cujos mais adquiridos são alimentos industrializados como salgadinhos, refrigerante e fast food. Esses alimentos são encontrados em toda parte, sempre acompanhados de muita propaganda, descontos e promoções, enquanto alimentos in natura ou minimamente processados nem sempre são comercializados em locais próximos aos centros urbanos. Por outro lado, os restaurantes são apontados como locais que possibilitam escolhas alimentares mais saudáveis, sendo que seus frequentadores possuem maior renda e média de escolaridade (BEZERRA IN, et al., 2013; BRASIL, 2014).

Outra forma mais recente de aquisição de alimentos é através dos Aplicativos de Delivery de Alimentos (ADAs). Devido à onipresença de smartphones e a conectividade de alta velocidade, no ano de 2017, o número de usuários de telefones celulares ultrapassaram cerca de cinco bilhões de indivíduos em todo o mundo. Além disso, o número de usuários de dispositivos móveis está cada vez maior, sendo que, através de seus telefones celulares ou tablets, conseguem pedir refeições e lanches por meio de aplicativos de delivery de alimentos, acionando seus restaurantes favoritos diretamente às suas portas com apenas alguns cliques. Por isso, as empresas de ADAs têm crescido e se tornaram a melhor plataforma para geração de aumento de receita (CHO M, et al., 2019).

Os ADAs são parte do ambiente digital da alimentação que servem como um canal mais conveniente para compras de alimentos online (GRANHEIM SI, 2019; PIGATTO G, et al., 2017). Mesmo com um preço maior devido às taxas de entrega, houve um aumento do uso de aplicativos devido a facilidade de seu uso entre os jovens (HENRIQUE DC, et al., 2020). Essa popularização dos deliverys ocorre pela falta de tempo devido ao estilo de vida e menor confiança e autonomia no preparo das refeições (MAIMAITI M, et al., 2018; BRASIL, 2014). Além disso, restaurantes que utilizam serviços de delivery possuem uma maior atratividade financeira e previsões otimistas devido à diminuição de gastos com recursos humanos e espaço físico (HENRIQUE DC, et al., 2020).

No Brasil, os ADAs estão presentes nos tablets e smartphones de $36 \%$ dos internautas brasileiros (IBOPE, 2017). Tais aplicativos utilizam estratégias como descontos e entregas gratuitas para o aumento das vendas, além de se comunicarem com seus consumidores através de e-mails, notificações dos aplicativos e propagandas nas redes sociais (YEO VCS, et al., 2017).

Dessa forma, o consumo de alimentos fora do domicílio está alterando cada vez mais os hábitos alimentares da população através da frequência do consumo de alimentos fora do lar, se mostrando 
positivamente associada ao maior o Índice de Massa Corpórea (IMC) da população (MCCRORY MA, et al.,2019). Além disso, a composição nutricional dos alimentos oferecidos nestes aplicativos é, na maioria das vezes, alta em calorias e baixa em nutrientes. Sendo assim, a compra de alimentos via aplicativos pode contribuir para um aumento do consumo de alimentos prejudiciais à saúde e para o desenvolvimento de doenças crônicas não transmissíveis (KEEBLE M, et al., 2020).

Portanto, este trabalho tem por objetivo avaliar o uso dos ADAs e a sua influência nos hábitos alimentares da população da Grande São Paulo.

\section{MÉTODOS}

Trata-se de um estudo observacional do tipo transversal, desenvolvido na Grande São Paulo, no período de 2019 e 2020. O estudo foi realizado com indivíduos, de ambos os sexos, com idade a partir de 18 anos. Participaram da pesquisa um total de 508 indivíduos, e a amostragem foi por conveniência. Foram excluídos do estudo os participantes que não conheciam e/ou faziam uso de aplicativo, não tiveram a anuência virtual do Termo de Consentimento Livre e Esclarecido e não residiam na região da grande São Paulo-SP ( $n=201$ ), totalizando uma amostra final de 307 pessoas.

O presente estudo foi aprovado pelo Comitê de Ética em Pesquisa (COEP) do Centro Universitário São Camilo (CAAE: 18841919.8.0000.0062).

\section{Procedimentos}

Os dados sobre o perfil dos participantes, o uso dos ADAs e sua correlação com os hábitos alimentares foram obtidos através da aplicação de questionário online elaborado previamente por meio do Google Forms ${ }^{\circledR}$. O questionário foi divulgado nas redes sociais dos autores através de um e-pôster e texto complementar para atrair os possíveis participantes, de dezembro de 2019 a janeiro de 2020. Ao final do questionário, os participantes recebiam orientação dos 10 Passos para Alimentação Adequada e Saudável do Guia Alimentar para População Brasileira, publicado pelo Ministério da Saúde (BRASIL, 2014).

\section{Critérios de avaliação e análise dos dados}

Para considerar e avaliar a influência do uso dos ADAs nos hábitos alimentares da população, foram desenvolvidos critérios. Os hábitos alimentares da população estudada foram avaliados através de um Questionário de Frequência Alimentar (QFA) baseado em uma lista pré-definida e adaptada de Fisberg RM, et al. (2008) e relacionados com o uso dos ADAs. Este instrumento foi utilizado para identificar o padrão alimentar dos participantes e associar tal padrão com o uso destes aplicativos.

O perfil socioeconômico da amostra foi distribuído de acordo com a frequência de utilização dos aplicativos e de cupons de desconto. Além disso, o questionário continha questões de autorrelato sobre a mudança dos hábitos alimentares dos participantes com o uso dos ADAs, com uma escala likert de 1 a 5 , representando "discordo totalmente" e "concordo totalmente", respectivamente. Este questionário foi utilizado para avaliar o perfil de utilização de ADAs da amostra a partir da autopercepção dos participantes.

Os dados do questionário foram analisados com o auxílio do programa Microsoft Office Excel $^{\circledR}$, versão 2016, sendo os resultados expostos em gráfico e tabelas. As análises estatísticas foram feitas com o auxílio do programa Statistical Package for the Social Sciences ${ }^{\circledR}$, versão 20.09 (SPSS, 2015). O valor de significância considerado foi de $p<0,05$.

\section{RESULTADOS E DISCUSSÃO}

O presente estudo identificou dados do perfil socioeconômico de 307 indivíduos, sendo 201 (65,5\%) do sexo feminino e 106 (34,5\%) do sexo masculino, com predominância entre 18 e 29 anos (53,4\%). Além disso, a maior parte da amostra possui ensino médio completo (45,9\%) e renda familiar maior que 6 salários-mínimos (SM) $(62,9 \%)$ (Tabela 1). 
Tabela 1 - Perfil socioeconômico dos participantes do estudo. São Paulo, 2021 ( $n=307)$.

\begin{tabular}{ll}
\hline Variáveis & $\mathbf{n}(\%)$ \\
\hline Sexo & \\
\hline Feminino & $201(65,5)$ \\
Masculino & $106(34,5)$ \\
\hline Idade & \\
\hline $18-24$ anos & $97(31,6)$ \\
$25-29$ anos & $67(21,8)$ \\
$30-39$ anos & $36(11,7)$ \\
$40-49$ anos & $35(11,4)$ \\
$50-59$ anos & $42(13,7)$ \\
60 ou mais & $30(9,8)$ \\
\hline Escolaridade & \\
\hline Ensino Fundamental Incompleto & $2(0,7)$ \\
Ensino Fundamental Completo & $18(5,8)$ \\
Ensino Médio Completo & $141(45,9)$ \\
Ensino Superior Completo & $50(16,3)$ \\
Pós Graduação Completa & $96(31,3)$ \\
\hline Renda Familiar & \\
\hline Até 2 SM & \\
Mais de 2 a 3 SM & $15(4,9)$ \\
Mais de 3 a 6 SM & $17(5,5)$ \\
Mais de 6 a 10 SM & $66(21,5)$ \\
Mais de 10 a 15 SM & \\
Mais de 15 SM & $53(17,3)$ \\
Sem Declaração & $42(13,7)$ \\
\hline
\end{tabular}

Legenda: *SM: Salário Mínimo.

Fonte: Alcantara FB, et al., 2021.

Pode-se confirmar que grande parte da população estudada apresenta consumo diário de pães, massas, arroz e outros cereais (76,0\%); carnes, leguminosas e/ou ovos $(77,2 \%)$; frutas legumes e verduras $(61,2 \%)$; e leites e derivados $(55,7 \%)$. Também foi identificado que o número de indivíduos que realizam o preparo das refeições diariamente é de $24,8 \%$.

O baixo nível de preparo diário das refeições pode estar relacionado com a praticidade que muitos lugares oferecem. Em concordância, o estudo de Jones SA, et al. (2014) relatou que através da disposição de cantinas universitárias, considerados locais convenientes e favoráveis na economia de tempo, o preparo de alimentos no ambiente doméstico se torna pouco atrativo.

Em relação ao perfil de utilização dos ADAs da população da Grande São Paulo, é possível observar que a maioria utiliza mais de um aplicativo $(70,7 \%)$, sendo eles, principalmente, o aplicativo A $(90,2 \%)$, seguido do aplicativo C $(47,2 \%)$ e do aplicativo B $(44,0 \%)$. Os ADAs são mais utilizados de 1 a 2 vezes por semana $(31,3 \%)$, principalmente de sábado $(71,3 \%)$, sexta-feira $(52,8 \%)$ ou domingo $(52,4 \%)$ e no período da noite, de $19 \mathrm{~h}-22 \mathrm{~h}(87,6 \%)$ e $23 \mathrm{~h}-2 \mathrm{~h}(17,9 \%)$.

Observa-se que a população do estudo utiliza os ADAs prioritariamente em suas casas $(92,8 \%)$, sendo o gasto mais comum entre 21 e 40 reais $(34,2 \%)$, para pedir pizza $(74,6 \%)$ e lanches $(60,3 \%)$, seguido de comida japonesa $(43,3 \%)$, árabe $(30,3 \%)$, brasileira (refeições caseiras) $(27,0 \%)$ e outros $(66,4 \%)$ (Tabela 2 ). 
Tabela 2 - Dados do perfil de utilização dos aplicativos de delivery de alimentos da população da Grande São Paulo. São Paulo, 2021.

\begin{tabular}{|c|c|}
\hline Variáveis & n (\%) \\
\hline \multicolumn{2}{|c|}{ Quantidade de aplicativos utilizados } \\
\hline Utilizam apenas 1 aplicativo & $90(29,3)$ \\
\hline Utilizam mais de 1 aplicativo & $217(70,7)$ \\
\hline \multicolumn{2}{|l|}{ Aplicativos utilizados } \\
\hline Aplicativo A & $277(90,2)$ \\
\hline Aplicativo B & $135(44,0)$ \\
\hline Aplicativo C & $145(47,2)$ \\
\hline Aplicativo D & $24(7,8)$ \\
\hline Outros & $97(31,6)$ \\
\hline \multicolumn{2}{|l|}{ Frequência de uso } \\
\hline Todos os dias & $3(1,0)$ \\
\hline 5-6 dias por semana & $4(1,3)$ \\
\hline 3-4 vezes por semana & $18(5,8)$ \\
\hline $1-2$ vezes por semana & $96(31,3)$ \\
\hline Quinzenalmente & $74(24,1)$ \\
\hline Mensalmente & $47(15,3)$ \\
\hline Raramente & $65(21,2)$ \\
\hline \multicolumn{2}{|l|}{ Dias de uso } \\
\hline Segunda-feira & $30(9,8)$ \\
\hline Terça-feira & $33(10,7)$ \\
\hline Quarta-feira & $42(13,7)$ \\
\hline Quinta-feira & $42(13,7)$ \\
\hline Sexta-feira & $162(52,8)$ \\
\hline Sábado & $219(71,3)$ \\
\hline Domingo & $161(52,4)$ \\
\hline \multicolumn{2}{|l|}{ Gasto médio por pedido } \\
\hline Menos de 10 reais & $0(0,0)$ \\
\hline $10-20$ reais & $18(5,8)$ \\
\hline $21-40$ reais & $105(34,2)$ \\
\hline $41-60$ reais & $72(23,5)$ \\
\hline $61-80$ reais & $45(14,7)$ \\
\hline $81-100$ reais & $40(13,0)$ \\
\hline Mais de 100 reais & $27(8,8)$ \\
\hline \multicolumn{2}{|l|}{ Local de utilização } \\
\hline Casa & $285(92,8)$ \\
\hline Trabalho & $49(16,0)$ \\
\hline Escola/Faculdade & $12(3,9)$ \\
\hline Outros & $25(8,1)$ \\
\hline \multicolumn{2}{|l|}{ Horário de utilização } \\
\hline $7 \mathrm{~h}-10 \mathrm{~h}$ & $9(2,9)$ \\
\hline $11 \mathrm{~h}-14 \mathrm{~h}$ & $46(15,0)$ \\
\hline $15 h-18 h$ & $19(6,2)$ \\
\hline $19 \mathrm{~h}-22 \mathrm{~h}$ & $269(87,6)$ \\
\hline $23 h-2 h$ & $55(17,9)$ \\
\hline \multicolumn{2}{|l|}{ Comida/alimentos pedido } \\
\hline Pizza & $229(74,6)$ \\
\hline Lanches & $185(60,3)$ \\
\hline Japonesa & $133(43,3)$ \\
\hline Brasileira (Refeições caseiras) & $83(27,0)$ \\
\hline Árabe & $93(30,3)$ \\
\hline Chinesa & $54(17,6)$ \\
\hline Saudável & $46(15,0)$ \\
\hline Outros & $204(66,4)$ \\
\hline
\end{tabular}

Fonte: Alcantara FB, et al., 2021. 
Os tipos de comida encontrados nos ADAs podem influenciar no aumento do consumo de alimentos ultraprocessados pela população brasileira. Horta PM, et al. (2020) destacaram a grande presença de alimentos ultraprocessados em geral nos cardápios disponíveis para os usuários dos aplicativos.

No estudo de Zion A, et al. (2019), que avaliou o perfil de uma amostra usuária de ADAs com idade maior de 18 anos, em um período de 90 dias, mostrou que os usuários de ADAs utilizaram os aplicativos de 1 a 2 vezes (48\%), 3 a 6 vezes (37\%), 7 a 10 vezes (9\%) e mais de 11 vezes (7\%). Tais resultados se revelam menores na frequência do uso dos aplicativos em comparação com os dados encontrados no presente estudo, confirmando a influência das diferentes características da população como as condições sócio-econômicas, culturais, políticas e, principalmente, o hábito alimentar divergente entre os povos.

A população do estudo acredita que pedir comida pelos ADAs não melhora a qualidade da sua alimentação $(61,6 \%)$, não aumenta o consumo de opções que eles consideram mais saudáveis $(64,1 \%)$ e que os alimentos pedidos não são mais saudáveis que a média da sua alimentação $(83,1 \%)$.

Aproximadamente metade da amostra $(49,2 \%)$ acredita que se não adquirissem comida pelos ADAs, a qualidade da refeição seria melhor. Aproximadamente a maioria concorda que o uso dos ADAs aumenta o consumo de fast food (48,5\%). No entanto, 40,7\% discordou sobre a influência destes aplicativos na quantidade de alimentos, no geral, consumidos habitualmente (Gráfico 1).

Gráfico 1 - Percepção da influência dos aplicativos de delivery de alimentos autorrelatada pela população da Grande São Paulo. São Paulo, 2021 ( $n=307$ ).

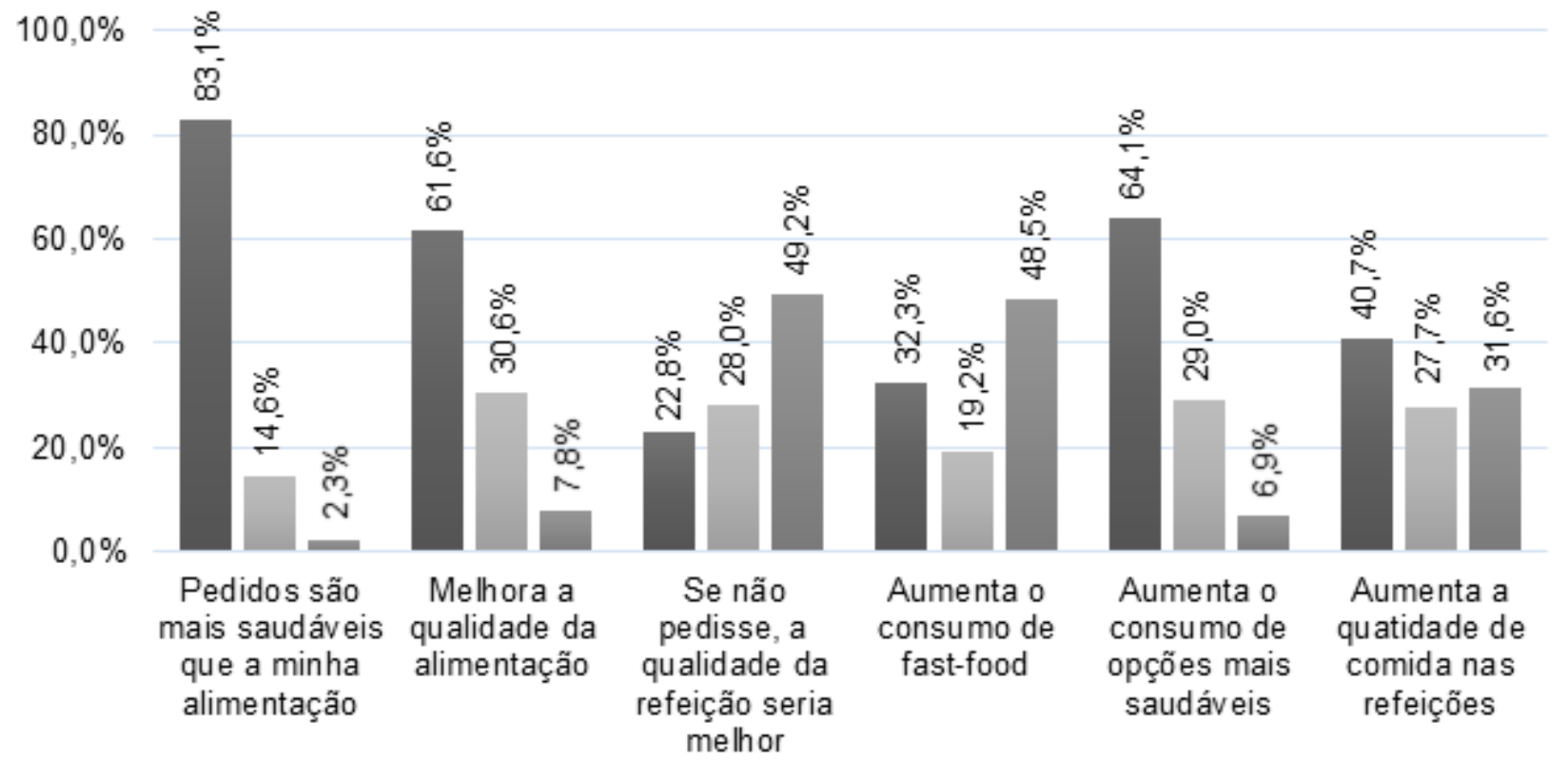

- Discordo Totalmente/Parcialmente $\backsim$ Não Discordo/Não Concordo n Concordo Parcialmente/Totalmente

Fonte: Alcantara FB, et al., 2021.

Segundo os relatos, observa-se que a maior forma de propaganda é por meio de notificações dos próprios ADAs (46,6\%). Além disso, os usuários consideram os ADAs o meio mais prático de se alimentar, independentemente da existência de cupom (33,0\%).

Por fim, constata-se que os cupons de desconto não exercem influência na decisão de compra dos usuários, pois seu recebimento é, principalmente, ocasional, segundo $26,4 \%$ da amostra. Além disso, $45,6 \%$ dos participantes referiram que, ao receber o cupom de desconto, não sentem vontade e não compram os alimentos pelos ADAs, e somente algumas compras são feitas com os cupons de desconto $(47,5 \%)$ (Tabela 3). 
Tabela 3 - Influência das propagandas dos aplicativos de delivery de alimentos nos hábitos alimentares da população da Grande São Paulo. São Paulo, 2021 ( $n=307)$.

\begin{tabular}{lc}
\hline Variáveis & $\mathbf{n}(\%)$ \\
\hline Formas de propaganda & \\
\hline Propaganda online & $133(43,3)$ \\
TV & $54(17,6)$ \\
Digital influencers & $22(7,2)$ \\
Cupons de desconto & $130(42,3)$ \\
E-mail & $61(19,9)$ \\
Mensagens via SMS & $129(42,0)$ \\
Notificações dos aplicativos & $143(46,6)$ \\
Outros & $23(7,5)$ \\
\hline Motivo principal para utilização do aplicativo & \\
\hline Praticidade & $101(33,0)$ \\
Fome & $32(10,4)$ \\
Desejo & $89(29,0)$ \\
Existência de cupom de desconto & $85(27,7)$ \\
\hline Frequência que recebem cupom de desconto & \\
\hline Nunca & $31(10,1)$ \\
Raramente & $48(15,6)$ \\
Ocasionalmente & $81(26,4)$ \\
Semanalmente & $70(22,8)$ \\
1 vez ao dia & $43(14,0)$ \\
2 vezes ao dia & $24(7,8)$ \\
3 ou mais vezes ao dia & $10(3,3)$ \\
\hline Utilização do cupom & \\
\hline Costumo sentir vontade de comprar e o utilizo no mesmo dia. & $31(10,1)$ \\
Costumo sentir vontade de comprar, mas não o utilizo. & $105(34,2)$ \\
Não me faz sentir vontade de comprar, mas costumo utilizá-lo no mesmo dia. & $31(10,1)$ \\
Não sinto vontade e nem compro. & $140(45,6)$ \\
\hline Compras realizadas utilizando o cupom & \\
\hline Todas & $15(4,9)$ \\
A maioria & $62(20,2)$ \\
Somente algumas & $146(47,5)$ \\
Nenhuma & $84(27,4)$ \\
\hline Fonte: Alcantara FB, et al., & \\
\hline
\end{tabular}

Fonte: Alcantara FB, et al., 2021.

Em concordância com os dados citados, o estudo de Maimaiti M et al. (2018) realizado com a população da China revelou que os principais motivos que os consumidores utilizam os ADAS são: quando se encontram em seus locais de trabalho (52,4\%), não querem sair de um ambiente para realizar as refeições $(51,4 \%)$, possuem pouco tempo/habilidade para cozinhar (39,8\%), presença de promoção (34,5\%), o clima/tempo está ruim $(26,0 \%)$, gostam do sabor da comida de delivery $(18,8 \%)$ ou faz parte do hábito alimentar $(12,9 \%)$.

Por outro lado, em um estudo realizado com a população indiana, a conveniência, juntamente com a pressão social, a experiência de entrega e o controle de qualidade não desempenharam um papel importante na intenção do uso dos aplicativos. Os fatores que tiveram uma associação significativa foram a experiência do cliente, a busca eficiente de lanchonetes e restaurantes famosos, a lista de restaurantes e opções de alimentos e a facilidade do uso dos aplicativos (RAY A, et al., 2019).

Assim, é possível afirmar que os motivos que levam às pessoas utilizarem os aplicativos variam de acordo com as características sócio-econômico cultural da população. Neste presente estudo, o principal motivo de utilização dos ADAs foi pela praticidade, não possuindo influência dos cupons de desconto.

Conforme os resultados analíticos sobre a relação entre a frequência de uso de cupons e perfil da população, observa-se que os resultados que obtiveram uma significância foram a relação com a idade $(p<0,001)$, renda familiar $(p<0,001)$, frequência do consumo de doces $(p=0,008)$ e fast food $(p=0,026)$, do recebimento de cupons $(p<0,001)$, gasto médio por pedido $(p<0,001)$, formas de propaganda dos aplicativos 
$(p<0,001)$ e o motivo do uso de cupons $(p<0,001)$ (Tabela 4). No entanto, alguns dados mostraram-se não significativos para este estudo, como a frequência do consumo de refrigerantes $(p=0,115)$, do preparo de refeições $(p=0,201)$ e do uso dos ADAs $(p=0,339)$.

Ao comparar a frequência do uso de cupons de desconto com a faixa etária, pode-se verificar que os jovens (18 a 29 anos) costumam utilizar mais cupons em suas compras $(p<0,001)$. Além disso, aqueles que possuem 3 a 10 SM $(p<0,001)$ e um gasto de $R \$ 21,00$ a $60,00(p<0,001)$ utilizam mais cupons nas compras (Tabela 4).

Em relação a frequência alimentar, o maior consumo de doces $(p=0,008)$ e fast food $(p=0,026)$ está associado a um maior uso de cupons de desconto. A frequência do consumo de refrigerantes $(p=0,115)$, preparo de refeições em casa $(p=0,201)$ e uso de ADAs $(p=0,339)$ não apresentaram significativa quando comparadas com a frequência do uso de cupons de desconto (Tabela 4). Além disso, ao comparar a frequência do uso de cupons de desconto com o motivo do uso de cupom, pode-se considerar que, independente da frequência de uso dos cupons, os usuários não costumam utilizar os cupons quando recebem $(p<0,001)$ (Tabela 4$)$.

Comparando os dados das tabelas, pode-se verificar que a maior parte da população do estudo $(79,8 \%)$ não utiliza cupom de desconto nas compras independente da vontade (Tabela 3). No entanto, os indivíduos que são influenciados por mais de um tipo de propaganda dos ADAs $(p<0,001)$ e recebem cupons com maior frequência $(p<0,001)$ costumam utilizá-los em maior proporção (Tabela 4$)$.

Tais dados revelam uma possível influência das formas de propagandas dos aplicativos na utilização de cupons de desconto. Sendo assim, corroboram com o estudo de Ray A, et al. (2019) que revelou que os mecanismos de engajamento dos consumidores como cupons de desconto, fidelização e propagandas são usados para aumentar o uso de ADAs.

Além do mais, a pesquisa de Nishiyama AF (2018) revelou que as notificações dos aplicativos geram um comportamento imediatista no consumidor, influenciando-o a tomar uma decisão e a agir de forma urgente. Sendo assim, tal imediatismo, quando usado no momento correto e somado à facilidade de compra dos aplicativos, permite uma quantidade maior de compras impulsivas, atendendo ao desejo momentâneo do consumidor.

Segundo o Guia Alimentar para a População Brasileira, o custo dos alimentos minimamente processados torna-se elevado quando comparados com os ultraprocessados. Além disso, há uma exposição populacional intensa à publicidade de alimentos não saudáveis que incentiva à população para o aumento do consumo de alimentos ultraprocessados (BRASIL, 2014). Sendo assim, considerando o resultado significante sobre a relação entre a frequência do uso de cupons e o aumento da frequência do consumo de doces e fast food, pode-se afirmar que, ao utilizar os cupons de desconto em suas compras, estes alimentos tornam-se mais vantajosos financeiramente em comparação aos alimentos minimamente processados.

Em relação aos resultados analíticos sobre a relação entre a frequência de uso de aplicativos e perfil da população, observa-se que os resultados que obtiveram uma significância foram a relação do uso de ADAs com a frequência do consumo de fast food $(p<0,001)$ e refrigerantes $(p<0,001)$, do preparo de refeições ( $p=0,046)$, o tipo de comida adquirido $(p<0,001)$, o motivo do uso de ADAs $(p<0,001)$, a frequência de recebimento de cupons $(p=0,004)$ e o autorrelato sobre a influência da qualidade dos alimentos disponíveis nos ADAS $(p<0,001)$ (Tabela 5). No entanto, alguns dados mostraram-se não significativos para este estudo, como a idade $(p=0,202)$, a frequência do consumo de doces $(p=0,172)$, aos tipos de propaganda recebidos $(p=0,314)$, a realização de compras utilizando cupons de desconto $(p=0,456)$ e o autorrelato sobre o uso dos ADAs aumentar o consumo de fast food $(p=0,121)$. 
Tabela 4 - Relação entre a frequência de uso de cupons e perfil da população do estudo. São Paulo, 2021 ( $n=307)$.

\begin{tabular}{|c|c|c|c|c|c|}
\hline Variáveis & Total & 3-7x/semana & 1-2x/semana & Ocasionalmente & $\mathbf{p}$ \\
\hline Idade n (\%) & & & & & $<0,001^{*}$ \\
\hline 18-24 anos & $97(31,6)$ & $40(51,9)$ & $47(32,2)$ & $10(11,9)$ & \\
\hline $25-29$ anos & $67(21,8)$ & $26(33,8)$ & $30(20,5)$ & $11(13,1)$ & \\
\hline 30-39 anos & $36(11,7)$ & $6(7,8)$ & $19(13,0)$ & $11(13,1)$ & \\
\hline $40-49$ anos & $35(11,4)$ & $3(3,9)$ & $11(7,5)$ & $21(25,0)$ & \\
\hline $50-59$ anos & $42(13,7)$ & $1(1,3)$ & $23(15,8)$ & $18(21,4)$ & \\
\hline 60 ou mais & $30(9,8)$ & $1(1,3)$ & $16(11,0)$ & $13(15,5)$ & \\
\hline Renda familiar n (\%) & & & & & $<0,001^{*}$ \\
\hline Até 2 SM & $15(4,9)$ & $5(6,5)$ & $7(4,8)$ & $3(3,6)$ & \\
\hline Mais de 2 a $3 \mathrm{SM}$ & $17(5,5)$ & $8(10,4)$ & $8(5,5)$ & $1(1,2)$ & \\
\hline Mais de 3 a 6 SM & $66(21,5)$ & $21(27,3)$ & $31(21,2)$ & $14(16,7)$ & \\
\hline Mais de 6 a 10 SM & $53(17,3)$ & $20(26,0)$ & $24(16,4)$ & $9(10,7)$ & \\
\hline Mais de 10 a 15 SM & $42(13,7)$ & $9(11,7)$ & $21(14,4)$ & $12(14,3)$ & \\
\hline Mais de $15 \mathrm{SM}$ & $98(31,9)$ & $5(6,5)$ & $54(37,0)$ & $39(46,4)$ & \\
\hline Sem declaração & $16(5,2)$ & $9(11,7)$ & $1(0,7)$ & $6(7,1)$ & \\
\hline Frequência de doces n (\%) & & & & & $0,008^{*}$ \\
\hline Diariamente & $95(30,9)$ & $28(36,4)$ & $44(30,1)$ & $23(27,4)$ & \\
\hline 3-5 vezes na semana & $96(31,6)$ & $26(33,8)$ & $48(32,9)$ & $22(26,2)$ & \\
\hline $1-2$ vezes na semana & $82(26,7)$ & $22(28,6)$ & $39(26,7)$ & $21(25,0)$ & \\
\hline Quinzenalmente & $14(4,6)$ & $1(1,3)$ & $8(5,5)$ & $5(6,0)$ & \\
\hline Raramente/ Nunca & $20(6,5)$ & 0 & $7(4,8)$ & $13(15,5)$ & \\
\hline Frequência de fast food n (\%) & & & & & $0,026^{*}$ \\
\hline Diariamente & 0 & 0 & 0 & 0 & \\
\hline 3-5 vezes na semana & $8(2,6)$ & $2(2,6)$ & $4(2,7)$ & $2(2,4)$ & \\
\hline $1-2$ vezes na semana & $90(29,3)$ & $26(33,8)$ & $45(30,8)$ & $19(22,6)$ & \\
\hline Quinzenalmente & $106(34,5)$ & $29(37,7)$ & $56(38,4)$ & $21(25,0)$ & \\
\hline Raramente/ Nunca & $103(33,6)$ & $20(26,0)$ & $41(28,1)$ & $42(50,0)$ & \\
\hline Frequência de refrigerantes $\mathrm{n}(\%)$ & & & & & 0,115 \\
\hline Diariamente & $18(5,9)$ & $2(2,6)$ & $6(4,1)$ & $10(11,9)$ & \\
\hline 3-5 vezes na semana & $23(7,5)$ & $7(9,1)$ & $13(8,9)$ & $3(3,6)$ & \\
\hline 1-2 vezes na semana & $65(21,2)$ & $16(20,8)$ & $36(24,7)$ & $13(15,5)$ & \\
\hline Quinzenalmente & $40(13,0)$ & $11(14,3)$ & $16(11,0)$ & $13(15,5)$ & \\
\hline Raramente/ Nunca & $161(52,4)$ & $41(53,2)$ & $75(51,4)$ & $45(53,6)$ & \\
\hline
\end{tabular}




\begin{tabular}{|c|c|c|c|c|c|}
\hline Variáveis & Total & 3-7x/semana & 1-2x/semana & Ocasionalmente & $\mathbf{p}$ \\
\hline Frequência de preparo $\mathrm{n}(\%)$ & & & & & 0,201 \\
\hline Diariamente & $76(24,8)$ & $19(24,7)$ & $31(21,2)$ & $26(31,0)$ & \\
\hline 3-5 vezes na semana & $80(26,1)$ & $23(29,9)$ & $35(24,0)$ & $22(26,2)$ & \\
\hline $1-2$ vezes na semana & $59(19,2)$ & $19(24,7)$ & $31(21,2)$ & $9(10,7)$ & \\
\hline Quinzenalmente & $25(8,1)$ & $4(5,2)$ & $15(10,3)$ & $6(7,1)$ & \\
\hline Raramente/ Nunca & $67(21,8)$ & $12(15,6)$ & $34(23,3)$ & $21(25,0)$ & \\
\hline Frequência de uso de aplicativos $\mathbf{n}(\%)$ & & & & & 0,339 \\
\hline Diariamente & $3(1,0)$ & $1(1,3)$ & $1(0,7)$ & $1(1,2)$ & \\
\hline 3-6 vezes na semana & $22(7,2)$ & $4(5,2)$ & $12(8,2)$ & $6(7,1)$ & \\
\hline $1-2$ vezes na semana & $96(31,3)$ & $25(32,5)$ & $50(34,2)$ & $21(25,0)$ & \\
\hline Quinzenalmente/ Mensalmente & $121(39,4)$ & $37(48,1)$ & $52(35,6)$ & $32(38,1)$ & \\
\hline Raramente & $65(21,2)$ & $10(13,0)$ & $31(21,2)$ & $24(28,6)$ & \\
\hline Gasto médio n (\%) & & & & & $<0,001^{*}$ \\
\hline $\mathrm{R} \$ 10,00-20,00$ & $18(5,9)$ & $13(16,9)$ & $1(0,7)$ & $4(4,8)$ & \\
\hline $\mathrm{R} \$ 21,00-40,00$ & $105(34,2)$ & $41(53,2)$ & $49(33,6)$ & $15(17,9)$ & \\
\hline $\mathrm{R} \$ 41,00-60,00$ & $72(23,5)$ & $16(20,8)$ & $40(27,4)$ & $16(19,0)$ & \\
\hline $\mathrm{R} \$ 61,00-80,00$ & $45(14,7)$ & $5(6,5)$ & $21(14,4)$ & $19(22,6)$ & \\
\hline Mais de $R \$ 80,00$ & $67(21,8)$ & $2(2,6)$ & $35(24,0)$ & $30(35,7)$ & \\
\hline Formas de propaganda $\mathrm{n}(\%)$ & & & & & $<0,001^{*}$ \\
\hline Propaganda online & $30(9,8)$ & $1(1,3)$ & $15(10,3)$ & $14(16,7)$ & \\
\hline Cupons de desconto & $10(3,3)$ & $5(6,5)$ & $5(3,4)$ & 0 & \\
\hline Notificações dos apps & $21(6,8)$ & $1(1,3)$ & $10(6,8)$ & $10(11,9)$ & \\
\hline Mensagens via SMS & $18(5,9)$ & $5(6,5)$ & $10(6,8)$ & $3(3,6)$ & \\
\hline Outros & $35(11,4)$ & $1(1,3)$ & $9(6,2)$ & $25(29,8)$ & \\
\hline Mais de 1 forma de propaganda & $193(62,9)$ & $64(83,1)$ & $97(66,4)$ & $32(38,1)$ & \\
\hline Frequência de recebimento de cupons $\mathrm{n}(\%)$ & & & & & $<0,001^{*}$ \\
\hline 3 ou mais vezes ao dia & $10(3,3)$ & $3(3,9)$ & $5(3,4)$ & $2(2,4)$ & \\
\hline $1-2$ vezes ao dia & $67(21,8)$ & $26(33,8)$ & $32(21,9)$ & $9(10,7)$ & \\
\hline Semanalmente & $70(22,8)$ & $27(35,1)$ & $35(24,0)$ & $8(9,5)$ & \\
\hline Ocasionalmente/ Raramente & $129(42,0)$ & $21(27,3)$ & $72(49,3)$ & $36(42,9)$ & \\
\hline Nunca & $31(10,1)$ & 0 & $2(1,4)$ & $29(34,5)$ & \\
\hline Motivo do uso do cupom (\%) & & & & & $<0,001^{*}$ \\
\hline Sente vontade e utiliza o cupom & $31(10,1)$ & $19(24,7)$ & $12(8,2)$ & 0 & \\
\hline Sente vontade e não utiliza o cupom & $105(34,2)$ & $39(50,6)$ & $52(35,6)$ & $14(16,7)$ & \\
\hline Não sente vontade, mas utiliza o cupom & $31(10,1)$ & $7(9,1)$ & $20(13,7)$ & $4(4,8)$ & \\
\hline Não sente vontade e nem utiliza o cupom & $140(45,6)$ & $12(15,6)$ & $62(42,5)$ & $66(78,6)$ & \\
\hline
\end{tabular}

Legenda: SM: Salário Mínimo. Fonte: Alcantara FB, et al., 2021. 
Tabela 5 - Relação entre a frequência de uso de aplicativos e perfil da população do estudo. São Paulo, 2021 ( $\mathrm{n}=307$ ).

\begin{tabular}{|c|c|c|c|c|c|}
\hline Variáveis & Total & 3-7x/semana & $1-2 x /$ semana & Ocasionalmente & $\mathbf{p}$ \\
\hline Idade n (\%) & & & & & 0,202 \\
\hline 18-24 anos & $97(31,6)$ & $6(24,0)$ & $27(28,1)$ & $64(34,4)$ & \\
\hline 25-29 anos & $67(21,8)$ & $6(24,0)$ & $21(21,9)$ & $40(21,5)$ & \\
\hline $30-39$ anos & $36(11,7)$ & $7(28,0)$ & $14(13,5)$ & $15(8,1)$ & \\
\hline $40-49$ anos & $35(11,4)$ & $3(12,0)$ & $13(14,6)$ & $19(10,2)$ & \\
\hline 50-59 anos & $42(13,7)$ & $1(4,0)$ & $14(14,6)$ & $27(14,5)$ & \\
\hline 60 ou mais & $30(9,8)$ & $2(8,0)$ & $7(7,3)$ & $21(11,3)$ & \\
\hline Frequência de doces n (\%) & & & & & 0,172 \\
\hline Diariamente & $95(30,9)$ & $12(48,0)$ & $34(35,4)$ & $49(26,3)$ & \\
\hline $3-5$ vezes na semana & $96(31,6)$ & $4(16,0)$ & $33(34,4)$ & $59(31,7)$ & \\
\hline $1-2$ vezes na semana & $82(26,7)$ & $6(24,0)$ & $17(17,7)$ & $59(31,7)$ & \\
\hline Quinzenalmente & $14(4,6)$ & $1(4,0)$ & $5(5,2)$ & $8(4,3)$ & \\
\hline Raramente/ Nunca & $20(6,5)$ & $2(8,0)$ & $7(7,3)$ & $11(5,9)$ & \\
\hline Frequência de fast food n (\%) & & & & & $<0,001^{*}$ \\
\hline $3-5$ vezes na semana & $8(2,6)$ & $6(24,0)$ & $1(1,0)$ & $1(0,5)$ & \\
\hline $1-2$ vezes na semana & $90(29,3)$ & $11(44,0)$ & $51(53,1)$ & $28(15,1)$ & \\
\hline Quinzenalmente & $106(34,5)$ & $2(8,0)$ & $18(18,8)$ & $86(46,2)$ & \\
\hline Raramente/ Nunca & $103(33,6)$ & $6(24,0)$ & $26(27,1)$ & $71(38,2)$ & \\
\hline Frequência de refrigerantes n (\%) & & & & & $<0,001^{*}$ \\
\hline Diariamente & $18(5,9)$ & $4(16,0)$ & $7(7,3)$ & $7(3,8)$ & \\
\hline $3-5$ vezes na semana & $23(7,5)$ & $5(20,0)$ & $12(12,5)$ & $6(3,2)$ & \\
\hline $1-2$ vezes na semana & $65(21,2)$ & $4(16,0)$ & $27(28,1)$ & $34(18,3)$ & \\
\hline Quinzenalmente & $40(13,0)$ & $1(4,0)$ & $10(10,4)$ & $29(15,6)$ & \\
\hline Raramente/ Nunca & $161(52,4)$ & $11(44,0)$ & $40(41,7)$ & $110(59,1)$ & \\
\hline Frequência de preparo $\mathrm{n}(\%)$ & & & & & $0,046^{*}$ \\
\hline Diariamente & $76(24,8)$ & $3(12,0)$ & $19(19,8)$ & $54(29,0)$ & \\
\hline $3-5$ vezes na semana & $80(26,1)$ & $3(12,0)$ & $28(29,2)$ & $49(26,3)$ & \\
\hline $1-2$ vezes na semana & $59(19,2)$ & $9(36,0)$ & $21(21,9)$ & $29(15,6)$ & \\
\hline Quinzenalmente & $25(8,1)$ & $4(16,0)$ & $10(10,4)$ & $11(5,9)$ & \\
\hline Raramente/ Nunca & $67(21,8)$ & $6(24,0)$ & $18(18,8)$ & $43(23,1)$ & \\
\hline Tipos de comida $\mathrm{n}(\%)$ & & & & & $<0,001^{*}$ \\
\hline Caseira & $4(1,3)$ & $2(8,0)$ & 0 & $2(1,1)$ & \\
\hline Fast food & $35(11,4)$ & 0 & $4(4,2)$ & $31(16,7)$ & \\
\hline Típicas & $4(1,3)$ & 0 & 0 & $4(2,2)$ & \\
\hline Outros & $5(1,6)$ & 0 & $4(4,2)$ & $1(0,5)$ & \\
\hline Mais que 1 opção & $259(84,4)$ & $23(92,0)$ & $88(91,7)$ & $148(79,6)$ & \\
\hline
\end{tabular}




\begin{tabular}{|c|c|c|c|c|c|}
\hline Variáveis & Total & 3-7x/semana & 1-2x/semana & Ocasionalmente & $\mathbf{p}$ \\
\hline Formas de propaganda $\mathrm{n}(\%)$ & & & & & 0,314 \\
\hline $\begin{array}{l}\text { Propaganda online } \\
\text { Cupons de desconto } \\
\text { Notificações dos apps } \\
\text { Mensagens via SMS } \\
\text { Outros } \\
\text { Mais de } 1 \text { forma de propaganda }\end{array}$ & $\begin{array}{c}30(9,8) \\
10(3,3) \\
21(6,8) \\
18(5,9) \\
35(11,4) \\
193(62,9) \\
\end{array}$ & $\begin{array}{c}2(8,0) \\
2(8,0) \\
3(12,0) \\
1(4,0) \\
2(8,0) \\
15(60,0)\end{array}$ & $\begin{aligned} & 13(13,5) \\
& 5(5,2) \\
& 8(8,3) \\
& 6(6,3) \\
& 7(7,3) \\
& 57(59,4) \\
&\end{aligned}$ & $\begin{array}{c}15(8,1) \\
3(1,6) \\
10(5,4) \\
11(5,9) \\
26(14,0) \\
121(65,1) \\
\end{array}$ & \\
\hline Motivo do uso de aplicativos $\mathrm{n}(\%)$ & & & & & $<0,001^{*}$ \\
\hline $\begin{array}{l}\text { Praticidade } \\
\text { Fome } \\
\text { Desejo } \\
\text { Existência de cupom de desconto }\end{array}$ & $\begin{array}{l}101(32,9) \\
32(10,4) \\
89(29,0) \\
85(27,7) \\
\end{array}$ & $\begin{array}{c}16(64,0) \\
1(4,0) \\
3(12,0) \\
5(20,0) \\
\end{array}$ & $\begin{array}{l}40(41,7) \\
13(13,5) \\
17(17,7) \\
26(27,1) \\
\end{array}$ & $\begin{array}{c}45(24,2) \\
18(9,7) \\
69(37,1) \\
54(29,0) \\
\end{array}$ & \\
\hline Frequência de recebimento de cupons $n(\%)$ & & & & & $0,004^{*}$ \\
\hline $\begin{array}{l}3 \text { ou mais vezes ao dia } \\
1-2 \text { vezes ao dia } \\
\text { Semanalmente } \\
\text { Ocasionalmente/ Raramente } \\
\text { Nunca }\end{array}$ & $\begin{array}{c}10(3,3) \\
67(21,8) \\
70(22,8) \\
129(42,0) \\
31(10,1) \\
\end{array}$ & $\begin{array}{c}0 \\
5(20,0) \\
7(28,0) \\
9(36,0) \\
4(16,0) \\
\end{array}$ & $\begin{array}{c}8(8,3) \\
24(25,0) \\
28(29,2) \\
29(30,2) \\
7(7,3) \\
\end{array}$ & $\begin{array}{c}2(1,1) \\
38(20,4) \\
35(18,8) \\
91(48,9) \\
20(10,8) \\
\end{array}$ & \\
\hline Compras utilizando cupons n (\%) & & & & & 0,456 \\
\hline $\begin{array}{l}\text { Todas as compras } \\
\text { A maioria } \\
\text { Somente algumas } \\
\text { Nenhuma }\end{array}$ & $\begin{array}{c}15(4,9) \\
62(20,2) \\
146(47,6) \\
84(27,4) \\
\end{array}$ & $\begin{array}{c}0 \\
5(20,0) \\
13(52,0) \\
7(28,0) \\
\end{array}$ & $\begin{array}{c}3(3,1) \\
22(22,9) \\
50(52,1) \\
21(21,9)\end{array}$ & $\begin{array}{c}12(6,5) \\
35(18,8) \\
83(44,6) \\
56(30,1)\end{array}$ & \\
\hline Alimentos pedidos são mais saudáveis que a minha alimentação $n(\%)$ & & & & & $0,001^{*}$ \\
\hline $\begin{array}{l}\text { Concordo } \\
\text { Não concordo nem discordo } \\
\text { Discordo }\end{array}$ & $\begin{array}{c}7(2,3) \\
45(14,7) \\
255(83,1) \\
\end{array}$ & $\begin{array}{c}1(4,0) \\
10(40,0) \\
14(56,0) \\
\end{array}$ & $\begin{array}{c}0 \\
11(11,5) \\
85(88,5) \\
\end{array}$ & $\begin{array}{c}6(3,2) \\
24(12,9) \\
156(83,9) \\
\end{array}$ & \\
\hline O uso de aplicativo aumenta o meu consumo de fast food $\mathrm{n}(\%)$ & & & & & 0,121 \\
\hline $\begin{array}{l}\text { Concordo } \\
\text { Não concordo nem discordo } \\
\text { Discordo }\end{array}$ & $\begin{array}{c}149(48,5) \\
59(19,2) \\
99(32,2) \\
\end{array}$ & $\begin{array}{l}12(48,0) \\
5(20,0) \\
8(32,0) \\
\end{array}$ & $\begin{array}{l}53(55,2) \\
10(10,4) \\
33(34,4) \\
\end{array}$ & $\begin{array}{l}84(45,2) \\
44(23,7) \\
58(31,2) \\
\end{array}$ & \\
\hline
\end{tabular}

Fonte: Alcantara FB, et al., 2021. 
Além disso, a maior frequência de preparo de alimentos em casa está relacionada com a menor frequência de uso dos ADAs $(p=0,046)$. Desta forma, estes dados corroboram com o fato de que a praticidade é o principal motivo daqueles que usam os ADAs com maior frequência $(p<0,001)$. Já a relação com a frequência do consumo de doces $(p=0,172)$, não revelou diferença significativa quando comparada com a frequência do uso de ADAs (Tabela 5).

Em relação ao grau de consciência da população do estudo, nota-se que os indivíduos que discordaram que os alimentos adquiridos são mais saudáveis que aqueles que consomem habitualmente usam os ADAs com menor frequência $(p<0,001)$. Entretanto, não houve diferença estatística entre a percepção do uso dos aplicativos aumentar o consumo de fast foods e a frequência de seu uso (Tabela 5).

Ao analisar a frequência de recebimento de cupons com a de uso de aplicativos, observa-se que quem recebe cupons ocasionalmente, utiliza os aplicativos ocasionalmente $(p=0,004)$. Todavia, não houve relação estatisticamente significativa entre a frequência de compras utilizando cupons $(p=0,456)$ e as formas de propaganda ( $p=0,314)$ com a frequência de uso dos ADAs (Tabela 5).

Sabe-se que a diferença de idade não teve relação estatística significativa com a frequência de uso dos ADAs $(p=0,202)$ (Tabela 5). Sendo assim, tal dado revela a facilidade de acesso aos ADAs de forma homogênea, ou seja, independente da faixa etária. Entretanto, a frequência do uso de cupons mostrou sofrer influência da idade $(p<0,001)$ (Tabela 4$)$.

Em contrapartida, o estudo de Zion A, et al. (2019) revelou que quanto menor a faixa etária, maior a frequência do uso dos aplicativos. Sendo $63 \%$ das pessoas com idade entre 18 a 29 anos, seguido por $51 \%$ entre 30 a 44 anos, 29\% entre 45 a 60 anos e somente 14\% acima de 60 anos. Portanto, estes dados confirmam que há uma diferença entre as culturas regionais estudadas, visto que a acessibilidade e a utilização dos ADAs se diferem, por múltiplos fatores, entre as duas populações demonstradas.

Os dados sobre alimentação revelam um maior número de indivíduos pedindo mais de um tipo de comida e uma maior frequência de consumo dos fast foods $(p<0,001)$ e refrigerantes $(p<0,001)$ dos indivíduos que utilizam os ADAs com maior frequência (Tabela 5). O alto consumo de fast food tem relação com o estudo de Bae YJ, et al. (2020), que revelou uma maior frequência do consumo de alimentos instantâneos nos indivíduos que utilizavam ADAs três ou mais vezes ao mês.

Dentre as limitações deste estudo, destaca-se o desenho transversal que não permite a avaliação temporal da população estudada. Os dados discutidos neste estudo não podem ser considerados para toda a população brasileira, visto que foi abordada somente uma região, desconsiderando as demais características regionais. Além disso, a literatura científica sobre este tema é escassa, portanto mais estudos são necessários para o melhor entendimento e discussão da influência do uso dos ADAs nos hábitos alimentares da população. Também, o trabalho, embora aborde temática importante para o campo da saúde coletiva, foi realizado em amostra de conveniência. Assim, os dados têm baixa generalização externa, não sendo adequados para realizar estimativas populacionais.

\section{CONCLUSÃO}

Considerando os resultados deste estudo, pode-se concluir que o uso dos ADAs independe das características socioeconômicas da população. Destaca-se que o uso dos aplicativos exerce uma influência negativa sobre os hábitos alimentares da amostra, em virtude das suas autopercepções e da utilização estar diretamente relacionada com o elevado consumo de fast food e refrigerantes, além de uma menor frequência de preparo das refeições em casa. Em relação ao uso de cupons de desconto, a maior parte da população do estudo não o utiliza quando recebe. No entanto, aqueles que recebem cupom com maior frequência e são influenciados por mais de um tipo de propaganda, os utilizam com uma periodicidade maior. Assim, o presente estudo contribui para entender como os ADAs estão influenciando a população e, principalmente, na elaboração de materiais e estratégias para melhorar os hábitos alimentares nessa nova realidade, visto que tais aplicativos surgiram após a publicação do nova Guia Alimentar para a População Brasileira. 


\section{REFERÊNCIAS}

1. BAE YJ, et al. Study on the Usage of Smartphone Food Delivery Apps among University Students in Chungbuk Area. Korean Journal of Food Nutrition, 2020; 33(1): 027-036.

2. BEZERRA IN, et al. Consumo de alimentos fora do domicílio no Brasil segundo locais de aquisição. Revista de Saúde Pública, 2013; 47: 200s-211s.

3. BRASIL. Ministério da Saúde. Guia Alimentar para a População Brasileira. 2nd ed. Brasília: MS; 2014.

4. BRASIL. Ministério da Saúde. Vigitel Brasil 2019: vigilância de fatores de risco e proteção para doenças crônicas por inquérito telefônico: estimativas sobre frequência e distribuição sociodemográfica de fatores de risco e proteção para doenças crônicas nas capitais dos 26 estados brasileiros e no Distrito Federal em 2019. Brasil: Ministério da Saúde. 2019.

5. $\mathrm{CHO} \mathrm{M}$, et al. Differences in perceptions about food delivery apps between single-person and multi-person households. International Journal of Hospitality Management, 2019; 77: 108-116.

6. FISBERG RM, et al. Questionário de freqüência alimentar para adultos com base em estudo populacional. Revista Saúde Pública, 2008; 42(3):550-4.

7. GRAHEIM SI. The digital food environment. UNSCN Nutrition 2019; 44:116-21.

8. HENRIQUE DC, et al. Foodservice e Aplicativos de Delivery: um estudo de viabilidade financeira em uma região universitária. Produto \& Produção, 2020; $21(2): 60-89$.

9. HORTA PM, et al. Digital food environment of a Brazil metropolis: food availability and marketing strategies used by delivery apps. Public Health Nutrition, 2020. 1-5.

10. IBGE. INSTITUTO BRASILEIRO DE GEOGRAFIA E ESTATÍstICA. Coordenação de Trabalho e Rendimento. Pesquisa de orçamentos familiares, 2017-2018: primeiros resultados. Rio de Janeiro: IBGE; 2019.

11. IBGE. INSTITUTO BRASILEIRO DE GEOGRAFIA E ESTATÍSTICA. Pesquisa de Orçamentos Familiares 2008-2009: despesas, rendimentos e condições de vida. Rio de Janeiro: IBGE; 2010.

12. IBOPE. INSTITUTO BRASILEIRO DE OPINIÃO PÚBLICA E ESTATÍSTICA. 2017. In: Um em cada três internautas tem app de delivery de comida. Disponível em: http://ibopeconecta.com/um-em-cada-tres-internautas-tem-app-dedelivery-de-comida/. Acesso em: 14 out. 2020.

13. JONES SA, et al. Perceived motivators to home food preparation: Focus group findings. J Acad Nutri Diet 2014; 114(10):1552-1556.

14. KEEBLE M, et al. Use of online food delivery services to order food prepared away-from-home and associated sociodemographic characteristics: a cross-sectional, multi-country analysis. Int J Environ Res Public Health 2020; 17:5190.

15. MAIMAITI M, et al. How we eat determines what we become: opportunities and challenges brought by food delivery industry in a changing world in China. European Journal of Clinical Nutrition, 2018; 72: 1282-1286.

16. MARTINS APB, et al. Increased contribuition of ultra-processed food products in the Brazilian diet (1987-2009). Rev Saúde Pública, 2013; 47: 1-10.

17. MCCRORY MA, et al. Fast-Food Offerings in the United States in 1986, 1991, and 2016 Show Large Increases in Food Variety, Portion Size, Dietary Energy, and Selected Micronutrients. Journal of the Academy of Nutrition and Dietetics, 2019; 119(6): 923-933.

18. NISHIYAMA AF. Arquiteturas da notícia em apps jornalísticos: características e tendências. Rev Famecos, 2018; 25(3): 1-13.

19. PIGATTO G, et al. Have you chosen your request? Analysis of online food delivery companies in Brazil. Br Food J, 2017; 119:639-57.

20. RAY A, et al. Why do people use food delivery apps (FDA)? A uses and gratification theory perspective. Journal of Retailing and Consumer Services, 2019; 51: 221-230.

21. SPSS Incorporation. Statistical package for the social science for windows student version/ SPSS [computer program] release 23.0 Chicago: marketing department, 2015.

22. YEO VCS, et al. Consumer experiences, attitude and behavioral intention toward online food delivery (OFD) services. Journal Retailing and Consumer Services, 2017; 35: 150-162.

23. ZION A, et al. 2019. Food Delivery Apps: Usage and Demographics - Winners, Losers and Laggards. Disponível em: https://www.zionandzion.com/research/food-delivery-apps-usage-and-demographics-winners-losers-andlaggards/. Acesso em: 14 out. 2020. 